\title{
Evaluation of Lateral Abdominal Muscle Activity during Expiratory Threshold Loading by Ultrasonography
}

\author{
Hideo KaneKo, PT, MS ${ }^{1)}$, Hironori SAto, $\mathrm{PhD}^{2)}$, Hitoshi Maruyama, PT, $\mathrm{PhD}^{3)}$ \\ 1) Department of Physical Therapy, School of Rehabilitation Sciences, International University \\ of Health and Welfare: 137-1 Enokizu, Ookawa City, Fukuoka 831-8501, Japan. \\ TEL+81944-89-2000 E-mail: hkaneko@iuhw.ac.jp \\ 2) Department of Health Science, Faculty of Applied Information Science, Hiroshima Institute \\ of Technology \\ ${ }^{3)}$ Department of Physical Therapy, School of Health Science, International University of \\ Health and Welfare
}

\begin{abstract}
We have examined whether the lateral abdominal muscle activity during expiratory loading can be noninvasively evaluated using ultrasonography. This study included 12 healthy adult men, and measured the thicknesses of their lateral abdominal muscles at the end of inspiration and the end of expiration, and the thickness differences when $5 \%, 10 \%$, and $15 \%$ of the maximal expiratory pressure were loaded as expiratory threshold loads. Tidal volume, inspiratory reserve volume and expiratory reserve volume were also measured at the same time. Obtained data were compared by expiratory threshold load, and correlation between muscle thickness and the thickness difference was assessed. Muscle thickness at the end of expiration and thickness difference significantly increased in proportion to the expiratory threshold load, and were accompanied by increase of tidal volume. Additionally, a significant positive correlation was found between muscle thickness and the thickness difference. The results of this study indicate that expiration activities of the lateral abdominal muscles can be evaluated by ultrasonography, and that the thickness difference reflects expiration activities.
\end{abstract}

Key words: Lateral abdominal muscles, Ultrasonography, Expiratory threshold loading

(This article was submitted Jul. 3, 2006 and was accepted Aug. 23, 2006)

\section{INTRODUCTION}

The abdominal muscles have important functions not only as flexors and rotators of the trunk, but also as also respiratory muscles. Among the abdominal muscles, the internal oblique muscle and the transversus abdominis muscle are closely related with the respiratory function. Especially, the transversus abdominis muscle works together with the diaphragm, and is closely related to the respiratory function. The respiratory functions of the abdominal muscles have been revealed by preceding electromyographic studies on the muscles under $\mathrm{CO}_{2}$ loading or expiratory threshold loading ${ }^{1-8)}$. In the preceding studies, needles or wire electrodes were used to evaluate the activities of inner muscles, such as the internal oblique muscle and the transversus abdominis muscle. A study evaluated patients with chronic obstructive pulmonary disease in a similar way, and reported transversus-abdominis-muscle activity in resting breathing 9). Such evaluations of respiratory functions of inner abdominal muscles are meaningful for further understanding of respiratory 
diseases. However, electromyographic evaluation with wire electrodes is an invasive method that imposes a burden on subjects, thus, it is difficult to use.

Recently, ultrasonography has drawn attention as a method that can noninvasively evaluate the activities of inner abdominal muscles ${ }^{10,11)}$. Several preceding studies have evaluated the function of abdominal muscles using ultrasonography equipment ${ }^{12-16)}$, but very few of them have evaluated the respiratory function. Misuri et al. ${ }^{12)}$ demonstrated the change of abdominal-muscle thickness in accordance with the position of lung volume and expiratory effort, and reported that ultrasonography is useful for evaluating the respiratory function of abdominal muscles, but no reported study has evaluated the activities of abdominal muscles using muscle thickness in relation to quantitative expiratory loads such as expiratory threshold load ${ }^{7,17)}$. Therefore, this study examined whether the muscle thickness measured by ultrasonography changes in proportion to the expiratory threshold load when the expiratory threshold load on healthy subjects is escalated.

\section{METHODS}

\section{Subjects}

The subjects of this study were 12 male students enrolled at a training institution for physical therapists with an average age of $28.6 \pm 4.4$ years, average height of $1.72 \pm 0.07 \mathrm{~m}$, average weight of $68.0 \pm 7.0 \mathrm{~kg}$, and average BMI of $22.8 \pm 1.3 \mathrm{~kg} / \mathrm{m}^{2}$ (mean \pm standard deviation). This study was approved by the ethical committee of the International University of Health and Welfare, and informed consent was obtained from the subjects following an explanation of the content of the study.

\section{Measurement of thickness of lateral abdominal muscles}

A B mode ultrasonograph SM-206 with a 3.5$\mathrm{MHz}$ linear probe (Seikousha Co. Ltd, Tokyo, Japan) was used to measure muscle thicknesses of the lateral abdominal muscles. The probe was placed transversely midway between the costal border and iliac crest on the right anterior axillary line. Then, ultrasonographic images of the external oblique muscle (EO), internal oblique muscle (IO), and transversus abdominis muscle (TA) were obtained $^{12)}$. Static images at the end of the inspiration and the end of expiration in 3 breathings were extracted from the ultrasonographic images recorded on a digital video. Scion Image (Scion Corporaiton, MD, USA) software was used for image analysis to measure the muscle thicknesses of EO, IO and TA, and the total of the 3 as the thickness of lateral abdominal muscles (LAB). Muscle thickness was measured in millimeters, and the mean of the 3 breaths was used as the representative value. The thickness difference (Tdif) was calculated as thickness at the end of expiration (Tee) minus thickness at the end of inspiration (Tei).

\section{Setting of expiration threshold load}

Threshold ${ }^{\circledR}$ inspiratory muscle trainer (Respironics Inc, PA, USA) was used as the ETL device. Expiratory resistance was generated by operating this device in the reverse direction. As a first step to setting an appropriate expiratory threshold load (ETL) in accordance with each subject's expiration ability, maximum expiratory pressure (PEmax) was measured with a mouth pressure meter MS-01 (Micromedical Technologies Inc., IL, USA) ${ }^{18)}$. The loads on the ETL device were set to $0 \%, 5 \%, 10 \%$ and $15 \%$ of PEmax.

\section{Spirometry during expiratory threshold loading}

The thickness of the abdominal muscles is affected by lung volume ${ }^{12)}$. Therefore, in order to measure lung volumes under ETL, a spirometer AS302 (Minato Medical Science Co. Ltd, Tokyo, Japan) was connected to Threshold IMT. Spirometry was performed to determine vital capacity (VC), tidal volume (TV), inspiratory reserve volume (IRV) and expiratory reserve volume (ERV) according to American Thoracic Society standards ${ }^{19)}$. In the spirometry under each ETL, results were valid only if the difference of VC from the value under $0 \%$ PEmax was within $5 \%$. If the difference was above $5 \%$, the spirometry was performed again.

\section{Procedure}

PEmax was measured with a subject sitting in an upright position on a chair with a nearly-vertical backrest. Subjects practiced breathing until the breathing became stable for each ETL (for about three minutes), then, muscle thicknesses of the lateral abdominal muscles and lung volumes were measured in order from $0 \%$ PEmax. The back of the 
subject was kept on the backrest during the measurement. Ultrasonographic images were recorded for 30 seconds when the breathing was stable, and the lung volumes were subsequently measured. All measurements were performed by the same investigator.

\section{Statistical analysis}

Means and standard deviations of muscle thicknesses of lateral abdominal muscles and lung volumes were calculated for each ETL. To compare muscle thicknesses and lung volumes among ETLs, one way repeated measures analysis of variance and Tukey's multiple comparison test were used. In addition, Pearson's correlation coefficient was calculated to assess the relation between Tee and Tdif. The level of significance was $5 \%$, and significance was defined as $\mathrm{p}<0.05$.

\section{RESULTS}

Tee significantly increased in proportion to ETL (Table 1). The differences were significant in LAB and TA under 5\% PEmax, IO under 10\% PEmax, and EO under 15\% PEmax. Additionally, LAB, $\mathrm{TA}$, and IO were significantly different between $5 \%$ PEmax and 15\% PEmax. Increase rates of Tee from $0 \%$ PEmax to $15 \%$ PEmax were $14 \%, 31 \%, 87 \%$ and $36 \%$ in $\mathrm{EO}$, IO, TA and LAB, respectively.

In contrast, there were no significant defference for Tei in any muscles (Table 2). Therefore, Tdif, similar to Tee and increased in proportion to ETL (Table 3). There were moderate or more significant positive correlations between Tee and Tdif (EO, $\mathrm{r}=0.67$; IO, $\mathrm{r}=0.68$; TA, $\mathrm{r}=0.88$; and $\mathrm{LAB}, \mathrm{r}=0.82$ ). However, Tdif under 5\% PEmax was significantly different only in LAB.

TV significantly increased under 5\% PEmax
(Table 4). It further increased under 15\% PEmax, but a significant difference was not seen $(5 \%$ PEmax vs. $15 \%$ PEmax, $\mathrm{p}=0.064 ; 10 \%$ PEmax vs. $15 \%$ PEmax, $\mathrm{p}=0.051)$. ERV significantly decreased under 15\% PEmax, but IRV showed no significant differences.

\section{DISCUSSION}

This study examined whether abdominal muscle thicknesses measured by ultrasonography changed in proportion to ETL. It was found that Tee increased in proportion to ETL. Especially, TA and LAB reflected changes in ETL well. Tdif also increased in proportion to ETL, indicating that it can be an indicator of expiration activities.

In preceding electromyographic studies of lateral abdominal muscles under expiratory loading, muscle activities increased in proportion to the expiratory load ${ }^{7,17)}$. In the present study, Tee of the lateral abdominal muscles also increased in proportion to ETL. In the lateral abdominal muscles, except EO, there were significant differences between 5\% PEmax and 15\% PEmax, which might be a result reflecting the expiration activities. In the preceding studies which used ETL, respiratory activities ${ }^{7)}$ and length ${ }^{3)}$ of lateral abdominal muscles were significantly shortened under ETL of 5-10 $\mathrm{cmH}_{2} \mathrm{O}$. Only TA and LAB showed significant difference under 5\% PEmax which was equivalent to the $5-10 \mathrm{cmH}_{2} \mathrm{O}$; IO and EO showed no significant differences under the same ETL. These results might be characteristics of the muscle activity evaluation using ultrasonography. In the measurement of muscle thickness by ultrasonography, the muscle activity of TA is more easily detected than that of IO, and that of EO is reportedly difficult to detect ${ }^{10}$. Therefore,

Table 1. Tee of lateral abdominal muscles during expiratory threshold loading $(\mathrm{mm})$

\begin{tabular}{lcccr}
\hline & $0 \%$ PEmax & $5 \%$ PEmax & $10 \%$ PEmax & $15 \%$ PEmax \\
\hline EO & $6.4 \pm 0.9$ & $6.6 \pm 1.4$ & $6.7 \pm 1.5$ & $7.3 \pm 1.6^{*}$ \\
IO & $10.2 \pm 1.8$ & $11.5 \pm 2.5$ & $13.0 \pm 3.1^{*}$ & $13.4 \pm 3.1^{* \dagger}$ \\
TA & $3.8 \pm 1.1$ & $5.2 \pm 1.5^{*}$ & $6.1 \pm 1.8^{*}$ & $7.1 \pm 1.6^{* \dagger}$ \\
LAB & $20.4 \pm 3.2$ & $23.3 \pm 4.3^{*}$ & $25.7 \pm 5.6^{*}$ & $27.8 \pm 5.5^{* \dagger}$ \\
\hline
\end{tabular}

Values are expressed as mean $\pm \mathrm{SD}$. LAB: lateral abdominal musckle; EO: external oblique musckle; IO: internal oblique musckle; TA: transversus abdominis musckle; Tee: thickness at end expiration. *: comparison with $0 \%$ PEmax $(p<0.05) .{ }^{\dagger}$ : comparison with 5\%PEmax $(p<0.05)$. 
Table 2. Tei of lateral abdominal muscles during expiratory threshold loading $(\mathrm{mm})$

\begin{tabular}{lrrrr}
\hline & 0\%PEmax & 5\%PEmax & 10\%PEmax & \multicolumn{1}{c}{$15 \%$ PEmax } \\
\hline EO & $5.9 \pm 1.0$ & $6.0 \pm 1.1$ & $5.9 \pm 1.0$ & $6.2 \pm 1.2$ \\
IO & $9.1 \pm 1.5$ & $9.0 \pm 2.2$ & $9.4 \pm 2.2$ & $9.7 \pm 2.6$ \\
TA & $3.1 \pm 0.7$ & $3.8 \pm 0.9$ & $3.9 \pm 1.1$ & $3.8 \pm 0.8$ \\
LAB & $18.1 \pm 2.7$ & $18.8 \pm 3.0$ & $19.2 \pm 3.4$ & $19.7 \pm 3.8$ \\
\hline
\end{tabular}

Values are expressed as mean $\pm \mathrm{SD}$. Tei: thickness at end inspiraiton.

Table 3. Tdif of lateral abdominal muscles during expiratory threshold loading $(\mathrm{mm})$

\begin{tabular}{lllll}
\hline & $0 \%$ PEmax & 5\%PEmax & $10 \%$ PEmax & $15 \%$ PEmax \\
\hline EO & $0.5 \pm 0.4$ & $0.6 \pm 0.5$ & $0.8 \pm 0.8$ & $1.1 \pm 0.9$ \\
IO & $1.2 \pm 0.7$ & $2.5 \pm 1.1$ & $3.6 \pm 1.8^{*}$ & $3.6 \pm 2.5^{* \dagger}$ \\
TA & $0.7 \pm 0.7$ & $1.4 \pm 1.6$ & $2.2 \pm 1.4^{*}$ & $3.3 \pm 1.4^{* \dagger}$ \\
LAB & $2.3 \pm 1.3$ & $4.6 \pm 2.5^{*}$ & $6.5 \pm 3.6^{*}$ & $8.0 \pm 3.6^{* \dagger}$ \\
\hline
\end{tabular}

Values are expressed as mean \pm SD. Tdif is the value of Tei subtracted from Tee. *: comparison with $0 \% \mathrm{PEmax}(\mathrm{p}<0.05) .{ }^{\dagger}$ : comparison with $5 \%$ PEmax $(\mathrm{p}<0.05)$.

Table 4. Expiratory threshold loading and lung volume

\begin{tabular}{lccll}
\hline & 0\%PEmax & 5\%PEmax & 10\%PEmax & 15\%PEmax \\
\hline ETL(cmH $\left.{ }_{2} \mathrm{O}\right)$ & 0 & $8.5 \pm 1.4$ & $17.0 \pm 2.6$ & $25.5 \pm 4.1$ \\
VC(L) & $4.64 \pm 0.54$ & $4.54 \pm 0.50$ & $4.53 \pm 0.50$ & $4.57 \pm 0.46$ \\
IRV(L) & $2.15 \pm 0.42$ & $1.92 \pm 0.41$ & $1.92 \pm 0.65$ & $1.86 \pm 0.66$ \\
TV(L) & $0.58 \pm 0.14$ & $0.93 \pm 0.26^{*}$ & $0.91 \pm 0.24^{*}$ & $1.29 \pm 0.58^{*}$ \\
ERV(L) & $1.91 \pm 0.29$ & $1.76 \pm 0.49$ & $1.73 \pm 0.60$ & $1.42 \pm 0.61^{*}$ \\
\hline
\end{tabular}

Values are expressed as mean \pm SD.VC: vital capacity; IRV: inspiratory reserve volume; TV: tidal vomume; ERV: expiratory reserve volume. *: comparison with $0 \%$ PEmax $(\mathrm{p}<0.05)$.

a higher ETL might have been necessary to see a significant increase of Tee in IO. However, since TA is most easily recruited against breathing load ${ }^{4,5)}$, TA was more likely to show a significant difference. Anyway, a clear increase of Tee in IO may suggest more forceful expiration than that in TA.

On the other hand, even in EO, whose activity is thought to be difficult to detect, Tee significantly increased under 15\% PEmax. This result was thought to be associated with the position of the lung volume, because ERV significantly decreased at the same time. In preceding studies, however, position of the lung volume was not associated with significant change of Tee in $\mathrm{EO}^{12)}$. Our result do not correspond to the results of preceding studies, but the change to occur is not known in the case of coincidence of position-of-lung-volume decrease and expiratory effort, and thus there is a possibility that Tee in EO was significantly increased by their synergy.

Tdif also increased in proportion to ETL. This was because Tei hardly changed even under ETL, and was also supported by the fact that Tdif and Tee were closely related. When estimating muscle activity based on Tee, the activity is usually estimated as the difference between 2 measurements under different conditions. Therefore, if lateral abdominal muscle activity is observed at rest, its activity cannot be estimated 
through muscle thickness. However, Tdif is the difference of muscle thicknesses accompanying breathing. Therefore, expiration activities can be estimated under one measurement condition. If expiration activities are enhanced even at rest, like in chronic obstructive pulmonary disease, Tdif could be used as an indicator. However, the difference in Tdif was small, and only LAB showed a significant difference under 5\% PEmax. Therefore, Tdif should be regarded as a change of all the lateral abdominal muscles. Additionally, since Tdif includes passive changes which do not reflect expiration activities ${ }^{3)}$, criteria to identify the enhancement of expiration activities are required. In this study, mean Tdif under 0\% PEmax was 2.3 $\mathrm{mm}$, but this result was obtained from breathing through the ETL device, and the subjects were only young men. In order to determine the enhancement of expiration activities, Tdif needs to be measured in wide variety of subjects.

\section{REFERENCES}

1) De Troyer A, Estenne M, Ninane V, et al.: Transversus abdominis muscle function in humans. J Appl Physiol, 1990, 68: 1010-1016.

2) Wakai Y, Welsh MM, Leevers AM, et al.: Expiratory muscle activity in the awake and sleeping human during lung inflation and hypercapnia. J Appl Physiol, 1992, 72: 881-887.

3) Leevers AM, Road JD: Abdominal muscle activation by expiratory threshold loading in awake dogs. Respir Physiol, 1993, 93: 289-303.

4) Abe T, Kusuhara N, Yoshimura N, et al.: Differential respiratory activity of four abdominal muscles in humans. J Appl Physiol, 1996, 80: 1379-1389.

5) Puckree T, Cerny F, Bishop B: Abdominal motor unit activity during respiratory and nonrespiratory tasks. $\mathrm{J}$ Appl Physiol, 1998, 84: 1707-1715.

6) Hodges PW, Heijnen I, Gandevia SC: Postural activity of the diaphragm is reduced in humans when respiratory demand increases. J Physiol, 2001, 537: 999-1008.

7) Yokoba M, Abe T, Katagiri M, et al.: Respiratory muscle electromyogram and mouth pressure during isometric contraction. Respir Physiol Neurobiol, 2003,
137: 51-60.

8) Hodges PW, Gandevia SC: Changes in intraabdominal pressure during postural and respiratory activation of the human diaphragm. J Appl Physiol, 2000, 89: 967-976.

9) Ninane V, Rypens F, Yernault JC, et al.: Abdominal muscle use during breathing in patients with chronic airflow obstruction. Am Rev Respir Dis, 1992, 146: $16-21$.

10) Hodges PW, Pengel LH, Herbert RD, et al.: Measurement of muscle contraction with ultrasound imaging. Muscle Nerve, 2003, 27: 682-692.

11) McMeeken JM, Beith ID, Newham DJ, et al.: The relationship between EMG and change in thickness of transversus abdominis. Clin Biomech, 2004, 19: 337342.

12) Misuri G, Colagrande S, Gorini $M$, et al.: In vivo ultrasound assessment of respiratory function of abdominal muscles in normal subjects. Eur Respir J, 1997, 10: 2861-2867.

13) Critchley DJ, Coutts FJ: Abdominal muscle function in chronic low back pain patients: measurement with real-time ultrasoud scanning. Physiotherapy, 2002, 8: 322-332.

14) Bo K, Sherburn M, Allen T: Transabdominal ultrasound measurement of pelvic floor muscle activity when activated directly or via a transversus abdominis muscle contraction. Neurourol Urodyn, 2003, 22: 582-588.

15) Ferreira PH, Ferreira ML, Hodges PW: Changes in recruitment of the abdominal muscles in people with low back pain: ultrasound measurement of muscle activity. Spine, 2004, 15: 2560-2566.

16) Ainscough-Potts AM, Morrissey MC, Critchley D: The response of the transverse abdominis and internal oblique muscles to different postures. Man Ther, 2006, 11: 54-60.

17) Barrett J, Cerny F, Hirsch JA, et al.: Control of breathing patterns and abdominal muscles during graded loads and tilt. J Appl Physiol, 1994, 76: 24732480.

18) American Thoracic Society/European Respiratory Society: ATS/ERS Statement on respiratory muscle testing. Am J Respir Crit Care Med, 2002, 166: 518624.

19) American Thoracic Society: Standardization of spirometry-1994 update. Am J Respir Crit Care Med, 1995, 152: 1107-1136. 